\title{
Long-term impact of 10 -valent pneumococcal conjugate vaccination on invasive pneumococcal disease among children in Finland
}

Hanna Rinta-Kokko ${ }^{\mathrm{a}}$, Arto A. Palmu ${ }^{\mathrm{b}}$, Kari Auranen ${ }^{\mathrm{c}}$, J. Pekka Nuorti ${ }^{\mathrm{d}, \mathrm{e}}$, Maija Toropainen ${ }^{\mathrm{d}}$, Lotta Siira $^{\mathrm{d}}$, Mikko J. Virtanen ${ }^{\mathrm{d}}$, Hanna Nohynek ${ }^{\mathrm{d}}$, Jukka Jokinen ${ }^{\mathrm{a}}$

\section{Affiliations}

${ }^{a}$ Department of Public Health Solutions, National Institute for Health and Welfare, P.O. Box 30, FI-00271 Helsinki, Finland

${ }^{\mathrm{b}}$ Department of Public Health Solutions, National Institute for Health and Welfare, Biokatu 10, FI33520 Tampere, Finland

${ }^{\mathrm{c}}$ Department of Mathematics and Statistics, University of Turku, 20014 Turun yliopisto, Turku, Finland

${ }^{\mathrm{d}}$ Department of Health Security, National Institute for Health and Welfare, P.O. Box 30, FI-00271 Helsinki, Finland

${ }^{\mathrm{e}}$ Department of Epidemiology, School of Health Sciences, University of Tampere, Kalevantie 4, FI33014, Tampere, Finland

\section{Corresponding author}

Hanna Rinta-Kokko, MSc

National Institute for Health and Welfare

Mannerheimintie 166, P.O. Box 30

00271 Helsinki, Finland

hanna.rinta-kokko@thl.fi

tel. +358295248141 


\begin{abstract}
Background

The ten-valent pneumococcal conjugate vaccine (PCV10) was introduced into the Finnish National Vaccination Programme (NVP) in September 2010. The impact of PCV10 vaccination against invasive pneumococcal disease (IPD) in vaccine-eligible children has been high, possibly due to cross-protection against PCV10-related serotypes (serotypes in the same serogroups as the PCV10 types) in addition to protection against PCV10 serotypes. We evaluated the long-term impact of PCV10 vaccination against IPD in vaccine-eligible and older, unvaccinated children six years after PCV10 introduction with a special focus on cross-protection.
\end{abstract}

\title{
Methods
}

We used data on IPD from the national, population-based surveillance. A target cohort of vaccineeligible children (born June 2010 or later) was followed from 3 months of age until the end of 2016. To assess the indirect effect, another cohort of older, PCV10-ineligible children was followed from 2012 through 2016. IPD rates were compared with those of season- and age-matched reference cohorts before NVP introduction.

\section{Results}

Among vaccine-eligible children, the incidence of all IPD decreased by 79\% (95\%CI 74 to $83 \%$ ). There was a statistically significant reduction in the incidence of 6A IPD, but for 19A, the reduction was non-significant and the incidence of 19A increased towards the end of the study period in the older vaccine-eligible children. The increase in non-PCV10 related serotypes was non-significant. 
In the unvaccinated older children, the incidence of all IPD decreased by 33\% (95\%CI 8 to $52 \%$ ) compared to the reference cohort. There was no impact on serotype 6A or 19A IPD in the unvaccinated cohort.

\section{Conclusion}

Overall, the impact of PCV10 vaccination on IPD was very high in vaccine-eligible children, with a major reduction in vaccine-type disease, and without notable replacement by other serotype groups. Our data suggest that PCV10 results in long-lasting direct cross-protection against 6A IPD. For 19A, no net reduction was observed six years after NVP introduction in the vaccine-eligible cohort. The indirect impact on IPD in unvaccinated children sustained. 
2 It has been estimated that Streptococcus pneumoniae (the pneumococcus) caused about 14.5 million

3 episodes of serious pneumococcal disease, including pneumonia, meningitis and febrile bacteraemia, worldwide in $2000 .{ }^{1}$ The introduction of pneumococcal conjugate vaccines (PCVs) since 2000 has afforded excellent direct protection for vaccinated children against invasive

6 pneumococcal disease (IPD) caused by serotypes included in the vaccines. At the same time, the

7 impact has extended to unvaccinated populations through indirect protection due to reduced

8 vaccine-type carriage in vaccinated children and the subsequently reduced transmission. However, replacement in carriage and subsequent disease by serotypes not included in the vaccines has partly eroded the direct and indirect benefits of vaccination across all age groups and is a growing

11 concern. $^{2-8}$

In addition to protection against the vaccine serotypes, PCVs have been documented to provide cross-protection against some vaccine-related serotypes, i.e. serotypes that belong to the same serogroups as the vaccine serotypes. Previous studies have shown that the seven-valent vaccine (PCV7, Prevenar, Pfizer), which included serotypes 4, 6B, 9V, 14, 18C, 19F, and 23F, reduced also IPD caused by 6A IPD, but not by 19A or 6C. ${ }^{3,9,10}$ Serotype 19A IPD was among the most common causes of replacement disease after PCV7 introduction., ${ }^{3,4,11,12}$ In 2010, PCV13 (Prevenar 13, Pfizer) with six additional serotypes (1, 3, 5, 6A, 7F, and 19A) replaced PCV7. Recently, cross-protection against $6 \mathrm{C}$ from the $6 \mathrm{~A}$ component of PCV13 has been reported ${ }^{13}$. The 10-valent pneumococcal conjugate vaccine (PCV10, Synflorix, GSK Vaccines) including serotypes in the PCV7 + 1, 5 and 7F was introduced into the Finnish National Vaccination Programme (NVP) after a public tender in September 2010. In addition to providing protection against vaccine serotypes, PCV10 also had reduced incidence of 6A and 19A IPD among vaccineeligible children in Finland three years after vaccine introduction into NVP. ${ }^{6}$ Here we report 
updated data about the overall and indirect long-term impact of PCV10 vaccination against IPD among vaccine-eligible and unvaccinated older children, with a special focus on cross-protection against the vaccine-related serotypes.

\section{Methods}

\section{The vaccination programme}

PCV10 was introduced into the NVP in September 2010. Children born on or after June 1, 2010, are eligible for vaccination with a $2+1$ schedule (primary series at 3 and 5 months and a booster dose at 12 months of age). There was no catch-up vaccination at PCV10 introduction. In the birth cohort of 2012, the uptake of at least one dose of PCV10 was estimated at 94\% based on data in the National Vaccination Register. ${ }^{14}$

In 2009-2011, the effectiveness of PCV10 against vaccine-type IPD was investigated in a nationwide FinIP vaccine trial, in which over 30,000 children born 2008 through May 2010 were vaccinated with PCV10. ${ }^{15}$ During the FinIP trial, the PCV10 vaccine coverage varied regionally from 0 to $60 \%$. Prior to the FinIP trial and the introduction of PCV10 into the NVP, no pneumococcal conjugate or polysaccharide vaccines were routinely used for healthy children and adults, and the vaccine uptake was estimated to be less than $2 \%$.

Influenza vaccine was introduced into the NVP in the beginning of the influenza season 2007/2008 for children aged 6 to 36 months. The uptake of this age group has been estimated to vary between 15 and $40 \% .^{14}$

\section{Data sources and case definition}

IPD cases were identified from the National Infectious Diseases Register (NIDR), a populationbased electronic laboratory surveillance system maintained by the National Institute for Health and 
Welfare (THL). It is mandatory for all clinical microbiology laboratories to notify all isolations of Streptococcus pneumoniae from blood or cerebrospinal fluid to NIDR and the process has been automated to send electronic reports to the database. Furthermore, the corresponding case isolates are sent to the national reference laboratory at THL for confirming the species and serotyping. Currently, more than $97 \%$ of the case isolates are received. ${ }^{16}$ IPD case was defined as isolation of $S$. pneumoniae from blood or cerebrospinal fluid. The IPD surveillance in Finland and the THL laboratory methods have been described earlier. ${ }^{6,17}$

Vaccination status of each IPD case was verified from the National Vaccination Register and local electronic vaccination cards. Data on comorbidities were obtained from the national hospital discharge register (the Care Register for Health Care at THL). The study population was determined by using data from the Finnish Population Information System. All register-based information was linked by using the unique national personal identity code which is assigned to all permanent residents in Finland.

IPD cases were categorized according to the causative serotype into three mutually exclusive groups: PCV10 serotypes, PCV10-related serotypes (i.e. serotypes belonging to the same serogroups as vaccine types; in the data: 6A, 6C, 7C, 9N, 18B, 19A, 23A, 23B), or non-PCV10 serotypes. The impact of PCV10 vaccination on each of the three serotype groups was evaluated. In addition, the impact was assessed separately for serotypes 6A and 19A.

\section{Overall impact of PCV10 vaccination in vaccine-eligible children}

To estimate the overall impact of PCV10 vaccination on IPD, the relative reduction in the incidence of IPD for each serotype group was estimated by comparing a target cohort, comprising all vaccineeligible children irrespective of vaccination status and born between June 2010 and September 2016, to a season- and age-matched reference cohort in years 2002-2008 (Figure 1, panel A). The 
children who were enrolled in the FinIP trial in years 2009-2010 were excluded from the analysis. The follow-up period started at 3 months of age (the first scheduled vaccination dose) and lasted until the end of December 2016 for the target cohort and December 2008 for the reference cohort. The follow-up thus included children from 3 to 78 months of age.

To assess time trends in the age-specific risk of 19A IPD, age-specific cumulative hazards were calculated for the target and reference cohorts.

\section{Indirect impact of PCV10 vaccination in unvaccinated children}

To estimate the indirect impact of PCV10 vaccination against IPD, the relative reduction in the incidence of IPD for each serotype group was estimated by comparison of unvaccinated cohorts of older children after and before PCV10 introduction (Figure 1, panel B). The unvaccinated target cohort was chosen to comprise children born between January 2006 and May 2010. Children vaccinated in the FinIP trial with PCV10 were excluded. The age- and season-matched reference cohort comprised all children born between January 2000 and May 2004. The follow-up period started in January 2012 (2006) and lasted until the end of December 2016 (2010) for the target (reference) cohort. The follow-up included children from 19 to 131 months of age.

\section{Statistical methods}

Comparison of IPD incidence rates was performed by using Poisson regression. Vaccine impact was defined as (1-incidence rate ratio)*100\%, comparing the target and reference cohorts. Absolute rate reductions and the corresponding confidence intervals were calculated from the parameter estimates with the delta method. No adjustments were made for comorbidities or influenza vaccinations in the NVP because of the small number of cases, evenly distributed comorbidities in the study cohorts and the low coverage of the influenza vaccinations. Statistical significance was deemed at the 5\% level. Statistical software R version 3.4.2 ${ }^{18}$ was used for all analyses. 
As part of its statutory tasks, the National Institute for Health and Welfare (THL) is obliged to monitor the effectiveness and safety of the vaccines used in NVP. The study plan was approved by the THL institutional review board (May 23, 2013). Permissions to use the register data for research were obtained from the relevant register controllers at THL (THL/1090/6.02.00/2013).

\section{Results}

\section{Overall impact of PCV10 vaccination in vaccine-eligible children}

Table 1 presents the estimated overall impact of PCV10 vaccination by the serotype groups, based on comparisons of the entire vaccine-eligible cohort (years 2010-2016) with its reference cohort (years 2002-2008). Among children targeted for vaccination, the incidence of all IPD decreased by $79 \%$ (95\% CI 74 to $83 \%$ ) from 42.9 to 9.2 cases per 100,000 person-years. The incidence of PCV10 serotype IPD decreased by $94 \%$ (95\% CI 91 to $96 \%$ ) from 32.3 to 1.9 cases per 100,000 personyears. There was a borderline non-significant increase in non-PCV10 serotypes (IRR $1.53,95 \% \mathrm{CI}$ 0.96 to 2.49). Figure 2 shows the evolution of the IPD incidence in children of vaccine-eligible age by epidemiological year (from July to June).

The incidence of IPD caused by PCV10-related serotypes decreased from 6.3 to 3.8 per 100,000 person-years. This was mainly due to a decrease in the incidence of $6 \mathrm{~A}$ with a relative reduction of $95 \%$ (95\% CI 75 to $100 \%$ ). There was a statistically non-significant reduction of $26 \%$ (95\% CI - 13 to $51 \%$ ) in $19 \mathrm{~A}$.

Apart from the notably high incidence in the epidemiological years 2003/2004-2004/2005, the incidence of 19A fluctuated over the years with some increase towards the end of the follow-up 
115 (Figure 3). The annual numbers of cases in 0-1 and 2-5 year-olds varied, respectively, between 0-17 and 0-12. Cases of 19A IPD appeared to occur more often among older children aged 24-78 months in the target cohort compared to the reference cohort (Figure 4, Supplement Figure 1). The presence of underlying comorbidities in 19A cases was low and similar in both cohorts. The serotypespecific changes in incidences are presented in Supplement Table 1.

There were six breakthrough cases defined as vaccine-type IPD more than two weeks after administration of the booster dose at 12 months among the fully vaccinated children (received all 3 doses). The causative serotypes were 19F ( 2 cases), 23F ( 2 cases), $6 \mathrm{~B}$ and 14 . No vaccine-type IPD cases occurred between two primary doses and a booster dose.

\section{Indirect impact of PCV10 vaccination in unvaccinated children}

Table 2 presents the relative and absolute rate reductions in IPD incidence, based on the unvaccinated target cohort of older children (19-131 months of age) during 2012-2016 and its reference cohort in the years 2006-2010. The incidence of PCV10 serotype IPD decreased by 58\% (95\% CI 37 to $73 \%$ ) from 5.9 cases in the reference cohort to 2.5 cases per 100,000 person-years in the target cohort, and the incidence of all IPD by 33\% (95\% CI 8 to 52\%) from 7.3 to 4.8 per 100,000 person-years (Figure 2). There was no indirect effect against the PCV10-related serotypes. The incidence of 19A IPD was 0.69 and 0.74 cases per 100,000 person-years, and that of 6A IPD 0.00 and 0.25 cases per 100,000 person-years in the reference and target cohorts, respectively (Figure 3, Supplement Table 2). The incidence of non-PCV10 serotypes was five times higher in the target-cohort compared to the reference cohort, but the confidence intervals were wide and the incidence remained low.

\section{Discussion}


137 In Finland, the introduction of PCV10 into the NVP has resulted in very high overall impact against IPD $(79 \%)$ in the vaccine-eligible birth cohorts six years after PCV10 implementation. This is explained by the almost complete elimination of PCV10-type and 6A IPD and the low incidence of other PCV10-related and non-PCV10 serotypes.

In unvaccinated older children, the indirect PCV10 impact on IPD was less notable (33\%) due to the smaller reduction in PCV10-serotype disease and small increases in PCV10-related and nonPCV10 disease.

According to a random-effect meta-analysis ${ }^{19}$, the pooled impact of PCV7 against all IPD was estimated to vary between 51 and $67 \%$ four to seven years after introduction among children $<5$ years of age. The impact of the combined PCV7 and PCV13 programmes against all IPD among children $<2$ years of age have been reported to vary between $64 \%$ (Stockholm, Sweden) to 89\% (Norway) $)^{7,20}$ and the combined PCV7 and PCV10 programmes among children $<5$ years of age from $64 \%$ (Quebec) to $80 \%$ (the Netherlands) ${ }^{5,21}$. Thus, our estimate of $79 \%$ is consistent with other settings.

Our data suggest that PCV10 results in long-lasting direct cross-protection against 6A IPD. In previous studies, the incidence of serotype 6A carriage and invasive disease were largely reduced in vaccinated children after PCV7 implementation, suggesting direct cross-protection from $6 \mathrm{~B}^{3,9,22}$, although not all studies have supported this. ${ }^{23}$ After PCV10 introductions, a non-significant direct cross-protection against 6A was found in Brazil $^{24}$ and Sweden ${ }^{13}$, and significant protection in Finland $^{6}$-- a finding that is further supported by the present study.

After PCV7 introduction in the USA, indirect cross-protection against 6A was observed among older children and adults ${ }^{9}$ although the effect was smaller and appeared later than the indirect impact against the PCV7 serotypes. ${ }^{11}$ In Sweden, a significant indirect cross-protection was 
reported after PCV10 vaccination in all unvaccinated age-groups. ${ }^{13}$ In our study, the number of cases of the older unvaccinated children was too small to assess indirect protection against 6A. However, 6A is not a replacing serotype among older children and adults in Finland in IPD $^{25}$ or in carriage in children ${ }^{26}$ suggesting some degree of indirect impact.

In contrast to $6 \mathrm{~A}$ and our earlier observation based on a three-year follow-up post introduction ${ }^{6}$, we observed no significant effect of PCV10 against 19A IPD. There were, however, differences by age and calendar time in 19A disease between the cohorts, as the cases of the vaccine-eligible cohort tended to be older and occur more often towards the end of the follow-up than those of the reference cohort. One explanation could be the increasing infection pressure from 19A, also seen in adult age groups 2012-2013 onwards ${ }^{25}$; another speculation is that the 19F component in PCV10 induces initial cross-protection which wanes with age thus rendering vaccinated children susceptible again to 19A in lack of specific 19A immunity. The small number of cases and year-to-year variability make firm conclusions difficult.

After PCV7 introduction in the USA and several countries in Europe, 19A carriage and the incidence of 19A IPD increased rapidly among children targeted to vaccination and also in older age groups. ${ }^{3,4,7,11,22}$ Our results are in contrast with the 3- to 4-fold increases of 19A IPD post-PCV7 in the USA in children ${ }^{11}$, although the increases in adults have been similar. ${ }^{11,25}$ In the US, the increase in 19A disease was mainly due to the increase in antimicrobial-resistant clonal complex (CC) 320 and a concurrent decrease in antimicrobial-susceptible CC199, suggesting that antibiotic selection pressure could have contributed to the 19A emergence ${ }^{27,28}$. In Finland, the outpatient antimicrobial use is lower than in $\mathrm{USA}^{29}$, and also lower than the average for all European Union countries $^{30}$, although higher compared to the Nordic neighbors. The introduction of PCV10 into the vaccination program has resulted in further reduction of antimicrobial use in children. ${ }^{31}$ The antimicrobial non-susceptibility of pneumococci has also decreased in all age-groups and especially 
among young children during the PCV10 program. ${ }^{32,33}$ For 19A, antimicrobial susceptible clones (especially ST994 and ST199) have become dominant both in children ${ }^{34}$ and among elderly people $^{35}$ during the post-PCV10 period in Finland resulting in low proportion of antimicrobial resistance.

In our first evaluation three years after PCV10 implementation in Finland, the 19A IPD incidence had declined significantly by $62 \%$ in the vaccine-eligible cohort (children aged 3-42 months). ${ }^{6}$ Statistically significant direct effectiveness against 19A IPD was reported with matched casecontrol studies in Brazil (82\%, children aged 2.6-53.1 months) and Quebec (79\%, children aged 259 months) after at least one PCV10 dose. ${ }^{24,36}$ In the Netherlands, where PCV10 was introduced about 5 years after PCV7 with a 3+1 schedule, the incidence of 19A IPD was lower in the PCV10eligible cohort than in the PCV7-eligible cohort. However, the cross-protective effect of 19A was inconclusive, as the incidence of both PCV10-related and non-related serotypes was low. ${ }^{5}$ In Sweden, where PCV7 was first introduced in the years 2007 through 2009 depending on the county and then replaced by PCV10 or PCV13 in 2009-2010, no direct cross-protection against 19A was reported in the PCV10 areas. ${ }^{13}$

The strengths of the current study setting include high data completeness $(\approx 80 \%$ of isolates in years 2002-2008 were available for serotyping, and $\approx 100 \%$ after that) and a well-established and consistent reporting in a population-based nationwide surveillance system. The personal identity code allowed linkage between different registers to find vaccination histories and health statuses for the IPD cases.

An apparent limitation of our study design, based on a before-after comparison, is its inclination to bias due to secular trends. In addition, the small numbers of cases caution against overinterpretation of analyses on individual serotypes. The age- and calendar-time related changes observed in 19A IPD may equally well be explained by chance alone: the annual numbers of 19A 
cases in under 2-year-olds in 2002-2016 ranged between 0 and 17, with median of 5 . Therefore, firm conclusions can only be made on the overall IPD level, where randomness and serotypespecific secular trends are balanced out with sufficient numbers and follow-up time. In addition, the vaccine-induced dynamics between serotypes in the post-vaccination period may limit comparisons with the reference cohort. Depending on the levels of competition between serotypes and vaccine coverage, replacement and herd protection in carriage and disease may occur differently. ${ }^{37}$

Moreover, influenza vaccinations that started in fall 2007 for children 6 to 36 months of age, may have prevented some IPD cases. However, the vaccination coverage was 40\% in 2007 to 2009 but less than $20 \%$ during the PCV10 program ${ }^{14}$, which might result in a conservative estimate of the PCV10 impact.

In conclusion, PCV10 vaccination has resulted in a very high impact against PCV10 serotypes, no notable replacement due to non-PCV10 serotypes, and long-lasting direct cross-protection against 6A IPD among vaccine-eligible children. Most importantly, the net impact on IPD irrespective of serotypes six years after PCV implementation in Finland is among the largest reported globally. $5,7,8,19$

\section{References}

1. World Health Organization (WHO). Immunization, vaccines and biologicals: Pneumococcal disease. http://www.who.int/immunization/topics/pneumococcal_disease/en/. Updated 2017. Accessed May/16, 2017. 
2. Whitney CG, Farley MM, Hadler J, et al. Decline in invasive pneumococcal disease after the introduction of protein-polysaccharide conjugate vaccine. N Engl J Med. 2003;348(18):1737-1746. doi: https://doi.org/10.1056/NEJMoa022823.

3. Hicks LA, Harrison LH, Flannery B, et al. Incidence of pneumococcal disease due to nonpneumococcal conjugate vaccine (PCV7) serotypes in the United States during the era of widespread PCV7 vaccination, 1998-2004. J Infect Dis. 2007;196(9):1346-1354.

4. Miller E, Andrews NJ, Waight PA, Slack MP, George RC. Herd immunity and serotype replacement 4 years after seven-valent pneumococcal conjugate vaccination in England and Wales: An observational cohort study. Lancet Infect Dis. 2011;11(10):760-768. doi: https://doi.org/10.1016/S1473-3099(11)70090-1.

5. Knol MJ, Wagenvoort GH, Sanders EA, et al. Invasive pneumococcal disease 3 years after introduction of 10-valent pneumococcal conjugate vaccine, the Netherlands. Emerg Infect Dis. 2015;21(11):2040-2044. doi: https://doi.org/10.3201/eid2111.140780.

6. Jokinen J, Rinta-Kokko H, Siira L, et al. Impact of ten-valent pneumococcal conjugate vaccination on invasive pneumococcal disease in Finnish children--a population-based study. PLoS One. 2015;10(3):e0120290. doi: https://doi.org/10.1371/journal.pone.0120290.

7. Steens A, Bergsaker MA, Aaberge IS, Ronning K, Vestrheim DF. Prompt effect of replacing the 7-valent pneumococcal conjugate vaccine with the 13-valent vaccine on the epidemiology of invasive pneumococcal disease in Norway. Vaccine. 2013;31(52):6232-6238. doi: https://doi.org/10.1016/j.vaccine.2013.10.032.

8. Waight PA, Andrews NJ, Ladhani SN, Sheppard CL, Slack MP, Miller E. Effect of the 13-valent pneumococcal conjugate vaccine on invasive pneumococcal disease in England and Wales 4 years 
251 after its introduction: An observational cohort study. Lancet Infect Dis. 2015;15(5):535-543. doi: serotypes 6A and 6C. J Infect Dis. 2008;198(12):1818-1822. doi: https://doi.org/10.1086/593339.

10. Choi YH, Jit M, Gay N, et al. 7-valent pneumococcal conjugate vaccination in England and Wales: Is it still beneficial despite high levels of serotype replacement? PlosOne. 2011;6(10):e26190.

11. Pilishvili T, Lexau C, Farley MM, et al. Sustained reductions in invasive pneumococcal disease in the era of conjugate vaccine. J Infect Dis. 2010;201(1):32-41. doi: serotype distribution and antimicrobial resistance of invasive Streptococcus Pneumoniae isolates in Dallas, TX, children from 1999 through 2005. Pediatr Infect Dis J. 2007;26(6):461-467. doi: https://doi.org/10.1097/INF.0b013e31805cdbeb. of the impact of pneumococcal conjugate vaccine 10 or pneumococcal conjugate vaccine 13 on 268 doi: 10.1093/cid/cix685 [doi].

269 14. Baum U, Sundman J, Jaaskelainen S, Nohynek H, Puumalainen T, Jokinen J. Establishing and 270 maintaining the national vaccination register in Finland. Euro Surveill. 2017;22(17):10.2807/1560271 7917.ES.2017.22.17.30520. doi: https://doi.org/10.2807/1560-7917.ES.2017.22.17.30520. 
15. Palmu AA, Jokinen J, Borys D, et al. Effectiveness of the ten-valent pneumococcal

273 Haemophilus Influenzae protein D conjugate vaccine (PHiD-CV10) against invasive pneumococcal 274 disease: A cluster randomised trial. Lancet. 2013;381(9862):214-222. doi:

275 https://doi.org/10.1016/S0140-6736(12)61854-6.

276 16. Siira L, Jalava J, Kaijalainen T, Ollgren J, Lyytikainen O, Virolainen A. Antimicrobial

277 resistance in relation to sero- and genotypes among invasive Streptococcus Pneumoniae in Finland, 278 2007-2011. Microb Drug Resist. 2014;20(2):124-130. doi: https://doi.org/10.1089/mdr.2013.0156.

279 17. Siira L, Kaijalainen T, Lambertsen L, Nahm MH, Toropainen M, Virolainen A. From Quellung 280 to multiplex PCR, and back when needed, in pneumococcal serotyping. J Clin Microbiol. 281 2012;50(8):2727-2731. doi: https://doi.org/10.1128/JCM.00689-12.

282 18. R Core Team (2013). R: A language and environment for statistical computing. R foundation 283 for statistical computing, Vienna, Austria. https://www.r-project.org/. Updated 2017. Accessed 284 May/16, 2017.

285 19. Feikin DR, Kagucia EW, Loo JD, et al. Serotype-specific changes in invasive pneumococcal 286 disease after pneumococcal conjugate vaccine introduction: A pooled analysis of multiple 287 surveillance sites. PLoS Med. 2013;10(9):e1001517. doi:

288 https://doi.org/10.1371/journal.pmed.1001517.

289 20. Galanis I, Lindstrand A, Darenberg J, et al. Effects of PCV7 and PCV13 on invasive 290 pneumococcal disease and carriage in Stockholm, Sweden. Eur Respir J. 2016;47(4):1208-1218. 291 doi: https://doi.org/10.1183/13993003.01451-2015. 
21. De Wals P, Lefebvre B, Markowski F, et al. Impact of $2+1$ pneumococcal conjugate vaccine program in the province of Quebec, Canada. Vaccine. 2014;32(13):1501-1506. doi: https://doi.org/10.1016/j.vaccine.2013.11.028.

22. Huang SS, Platt R, Rifas-Shiman SL, Pelton SI, Goldmann D, Finkelstein JA. Post-PCV7 changes in colonizing pneumococcal serotypes in 16 Massachusetts communities, 2001 and 2004. Pediatrics. 2005;116(3):e408-13.

23. Kellner JD, Scheifele D, Vanderkooi OG, Macdonald J, Church DL, Tyrrell GJ. Effects of routine infant vaccination with the 7 -valent pneumococcal conjugate vaccine on nasopharyngeal colonization with Streptococcus Pneumoniae in children in Calgary, Canada. Pediatr Infect Dis J. 2008;27(6):526-532. doi: https://doi.org/10.1097/INF.0b013e3181658c5c.

24. Domingues CM, Verani JR, Montenegro Renoiner EI, et al. Effectiveness of ten-valent pneumococcal conjugate vaccine against invasive pneumococcal disease in Brazil: A matched casecontrol study. Lancet Respir Med. 2014;2(6):464-471. doi: https://doi.org/10.1016/S22132600(14)70060-8.

25. Nuorti JP, Rinta-Kokko H, Toropainen M, et al. Evidence of herd protection and serotype replacement in adults after universal 10-valent pneumococcal conjugate vaccination of infants in Finland. 2016;10th International Symposium on Pneumococci and Pneumococcal Diseases (ISPPD10), Glasgow, Scotland.

26. Palmu AA, Toropainen M, Kaijalainen T, et al. Direct and indirect effectiveness of the 10valent pneumococcal conjugate vaccine against carriage in a cluster randomized trial. Pediatr Infect Dis J. 2017;36(12):1193-1200. doi: https://doi.org/10.1097/INF.0000000000001705. 

321 [pii].

27. Van Effelterre T, Moore M, Fierensa F, et al. A dynamic model of pneumococcal infection in the United States: Implications for prevention through vaccination. Vaccine. 2010;28:3650-3660.

28. Beall BW, Gertz RE, Hulkower RL, Whitney CG, Moore MR, Brueggemann AB. Shifting genetic structure of invasive serotype 19A pneumococci in the United States. J Infect Dis. 2011;203(10):1360-1368. doi: 10.1093/infdis/jir052 [doi].

29. Goossens H, Ferech M, Coenen S, Stephens P, European Surveillance of Antimicrobial Consumption Project Group. Comparison of outpatient systemic antibacterial use in 2004 in the United States and 27 European countries. Clin Infect Dis. 2007;44(8):1091-1095. doi: CID41134

30. European Centre for Disease Prevention and Control, ECDC. Summary of the latest data on antibiotic consumption in the European Union.

https://ecdc.europa.eu/sites/portal/files/documents/antibiotics-consumption-EU-data-2014.pdf. Updated 2014. Accessed 01/26, 2018.

31. Palmu AA, Rinta-Kokko H, Nohynek H, Nuorti JP, Jokinen J. Impact of national ten-valent pneumococcal conjugate vaccine program on reducing antimicrobial use and tympanostomy tube placements in Finland. Pediatr Infect Dis J. 2018;37(1):97-102. doi:

10.1097/INF.0000000000001810 [doi].

32. Siira L, Toropainen M, Nohynek H, Nuorti JP, Jalava J. Changes in invasive pneumococcal serotypes and antimicrobial susceptibility before and after PCV10 introduction in Finland, 20052014. 2016;10th International Symposium on Pneumococci and Pneumococcal Diseases, Glasgow, Scotland. 
334 33. Jalava J, Räisänen K(). Bakteerien mikrobilääkeresistenssi Suomessa. 2016;National Institute 335 for Health and Welfare, Finland. In Finnish.

336 34. Toropainen M, Siira L, Jalava J, Palmu AA, Rinta-Kokko H, Nuorti JP. Molecular 337 epidemiology and antimicrobial susceptibility of invasive serotype 19A Streptococcus Pneumoniae 338 in Finnish children, 2007-2015. 2016;10th International Symposium on Pneumococci and 339 Pneumococcal diseases, Glasgow, Scotland.

340 35. Toropainen M. Unpublished observation. 2018.

341 36. Deceuninck G, De Serres G, Boulianne N, Lefebvre B, De Wals P. Effectiveness of three 342 pneumococcal conjugate vaccines to prevent invasive pneumococcal disease in Quebec, Canada. 343 Vaccine. 2015;33(23):2684-2689. doi: https://doi.org/10.1016/j.vaccine.2015.04.005.

344 37. Lipsitch M. Vaccination against colonizing bacteria with multiple serotypes. Proc Natl Acad Sci $345 \quad U S$ A. 1997;94(12):6571-6576. 
349 National Institute for Health and Welfare has received research funding from GlaxoSmithKline 350 Vaccines for the conduct of a nationwide effectiveness trial of the 10-valent pneumococcal 351 conjugate vaccine. HR-K, AAP, MT, LS, and JJ are co-investigators in these studies. The other 352 authors have no conflicts to disclose. Current study was entirely publicly funded.

\section{Authorship contributions}

354 HR-K coordinated the data collection, carried out the analyses, interpreted the data and drafted the 355 manuscript; AAP, KA, JPN, HN, and JJ designed the study, interpreted the data and reviewed and 356 revised the manuscript; MT, LS, and MJV supervised the microbiological data quality and reviewed 357 and revised the manuscript; and all authors approved the final manuscript as submitted.

358 
361 Table 1. Incidence rates of invasive pneumococcal disease (IPD) and the corresponding relative and 362 absolute incidence rate reductions, based on the comparison of the PCV10-eligible target cohort 363 (2010-2016) vs. a reference cohort (2002-2008).

364

Table 2. Incidence rates of invasive pneumococcal disease (IPD) and the corresponding relative and absolute incidence rate reductions, based on the comparison of an unvaccinated target cohort (20122016) vs. a reference cohort (2006-2010).

Figure 1. Target and reference cohorts for estimating the overall impact of PCV10 on IPD among vaccine-eligible children (panel A) and unvaccinated children (panel B).

Figure 2. Incidence rates of invasive pneumococcal disease (IPD) in age groups $<2$ years (panel A) and 2-5 years (panel B) by serotype group in epidemiological years 6/2002-6/2017.

Figure 3. Incidence rates of 19A and 6A IPD in age groups <2 years (panel A) and 2-5 years (panel B) in epidemiological years 6/2002-6/2017.

Figure 4. Incidence of all IPD (first panel) and 19A IPD (second panel) in the PCV10-eligible target and reference cohorts by age group. 
382 Supplement table 1. Incidence rates of invasive pneumococcal disease (IPD) of individual PCV10 383 serotypes and the corresponding relative and absolute incidence rate reductions, based on the 384 comparison of the PCV10-eligible target cohort (2010-2016) vs. a reference cohort (2002-2008). 385

386 Supplement table 2. Incidence rates of invasive pneumococcal disease (IPD) of individual PCV10 387 serotypes and the corresponding relative and absolute incidence rate reductions, based on the 388 comparison of an unvaccinated target cohort (2012-2016) vs. a reference cohort (2006-2010). 389

390 Supplement Figure 1. Age-specific cumulative hazards of 19A IPD in the PCV10-eligible target 391 cohort and the reference cohort. 
Table 1. Incidence rates of invasive pneumococcal disease (IPD) and the corresponding relative and absolute incidence rate reductions, based on the comparison of the PCV10-eligible target cohort (2010-2016) vs. a reference cohort (2002-2008).

Table 2. Incidence rates of invasive pneumococcal disease (IPD) and the corresponding relative and absolute incidence rate reductions, based on the comparison of an unvaccinated target cohort (20122016) vs. a reference cohort (2006-2010).

Table 1.

\begin{tabular}{|c|c|c|c|c|}
\hline & \multicolumn{2}{|c|}{ Incidence per 100,000 person-years $(\mathrm{N})$} & \multicolumn{2}{|c|}{ Target cohort vs. reference cohort } \\
\hline & $\begin{array}{l}\text { Reference cohort } \\
\text { 2002-2008* } \\
\text { Follow-up yrs } \\
1211504 \\
\end{array}$ & $\begin{array}{l}\text { Target cohort } \\
\text { 2010-2016** } \\
\text { Follow-up yrs } \\
1243145 \\
\end{array}$ & $\begin{array}{l}\text { Relative rate reduction, \% } \\
(95 \% \mathrm{CI})\end{array}$ & $\begin{array}{l}\text { Absolute rate reduction per } \\
100,000 \text { person-years }(95 \% \mathrm{Cl})\end{array}$ \\
\hline Any culture confirmed IPD & $42.9(520)$ & $9.2(114)$ & $78.6(73.9,82.6)$ & $33.8(29.7,37.8)$ \\
\hline PCV10 serotypes & $32.3(391)$ & $1.9(23)$ & $94.3(91.5,96.3)$ & $30.4(27.1,33.7)$ \\
\hline PCV10-related serotypes & $6.3(76)$ & $3.8(47)$ & $39.7(13.7,58.4)$ & $2.5(0.72,4.3)$ \\
\hline Non-PCV10 serotypes & $2.3(28)$ & $3.5(44)$ & $-53.1(-148.6,4.1)$ & $-1.2(-2.6,0.12)$ \\
\hline Not known & $2.1(25)$ & $0(0)$ & NA & NA \\
\hline
\end{tabular}

*Age 3-78 months, born Jun 2002 - Sep 2008

**Age 3-78 months, born Jun 2010 - Sep 2016

$\mathrm{NA}=$ not applicable

Table 2.

\begin{tabular}{|c|c|c|c|c|}
\hline \multirow[t]{2}{*}{ Serotype group } & \multicolumn{2}{|c|}{ Incidence per 100,000 person-years $(N)$} & \multicolumn{2}{|c|}{ Unvaccinated target cohort vs. reference cohort } \\
\hline & $\begin{array}{l}\text { Reference cohort } \\
\text { 2006-2010* } \\
\text { Follow-up yrs } \\
1309618\end{array}$ & $\begin{array}{l}\text { Target cohort } \\
\text { 2012-2016** } \\
\text { Follow-up yrs } \\
1221933\end{array}$ & $\begin{array}{l}\text { Relative rate reduction, \% } \\
(95 \% \mathrm{Cl})\end{array}$ & $\begin{array}{l}\text { Absolute rate reduction per } \\
100,000 \text { person-years }(95 \% \mathrm{Cl})\end{array}$ \\
\hline Any culture confirmed IPD & $7.3(95)$ & $4.8(59)$ & $33.4(8.2,52.1)$ & $2.4(0.52,4.3)$ \\
\hline PCV10-serotypes & $5.9(77)$ & $2.5(30)$ & $58.2(37.1,73.0)$ & $3.4(1.8,5)$ \\
\hline PCV10-related serotypes & $0.69(9)$ & $1.2(15)$ & $-78.6(-325.3,20.5)$ & $-0.54(-1.3,0.23)$ \\
\hline Non-PCV10 serotypes & $0.23(3)$ & $1.2(14)$ & $-400.2(-2069.8,-63.3)$ & $-0.92(-1.8,-0.26)$ \\
\hline Not known & $0.46(6)$ & $0(0)$ & NA & NA \\
\hline
\end{tabular}

*Age 19-131 months, born Jan 2000 - May 2004

**Age 19-131 months, born Jan 2006 - May 2010

$\mathrm{NA}=$ not applicable 

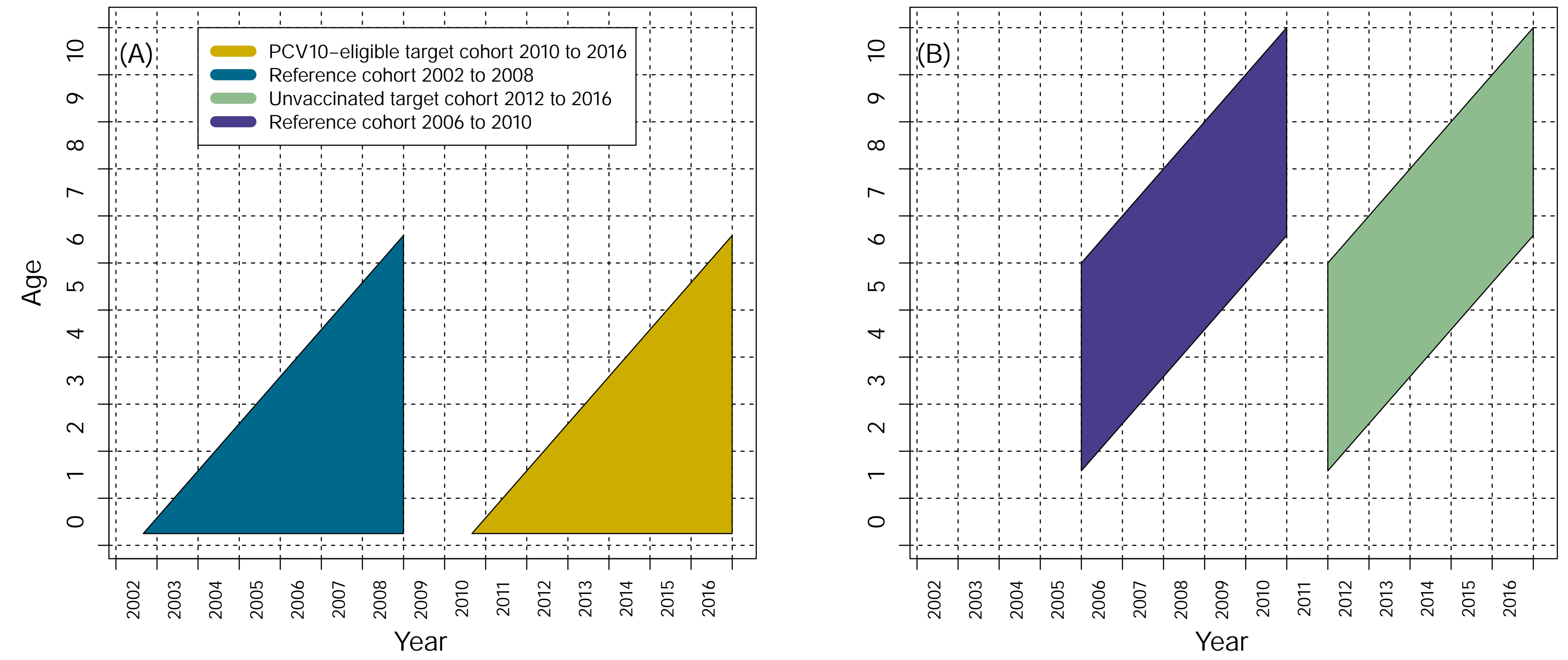


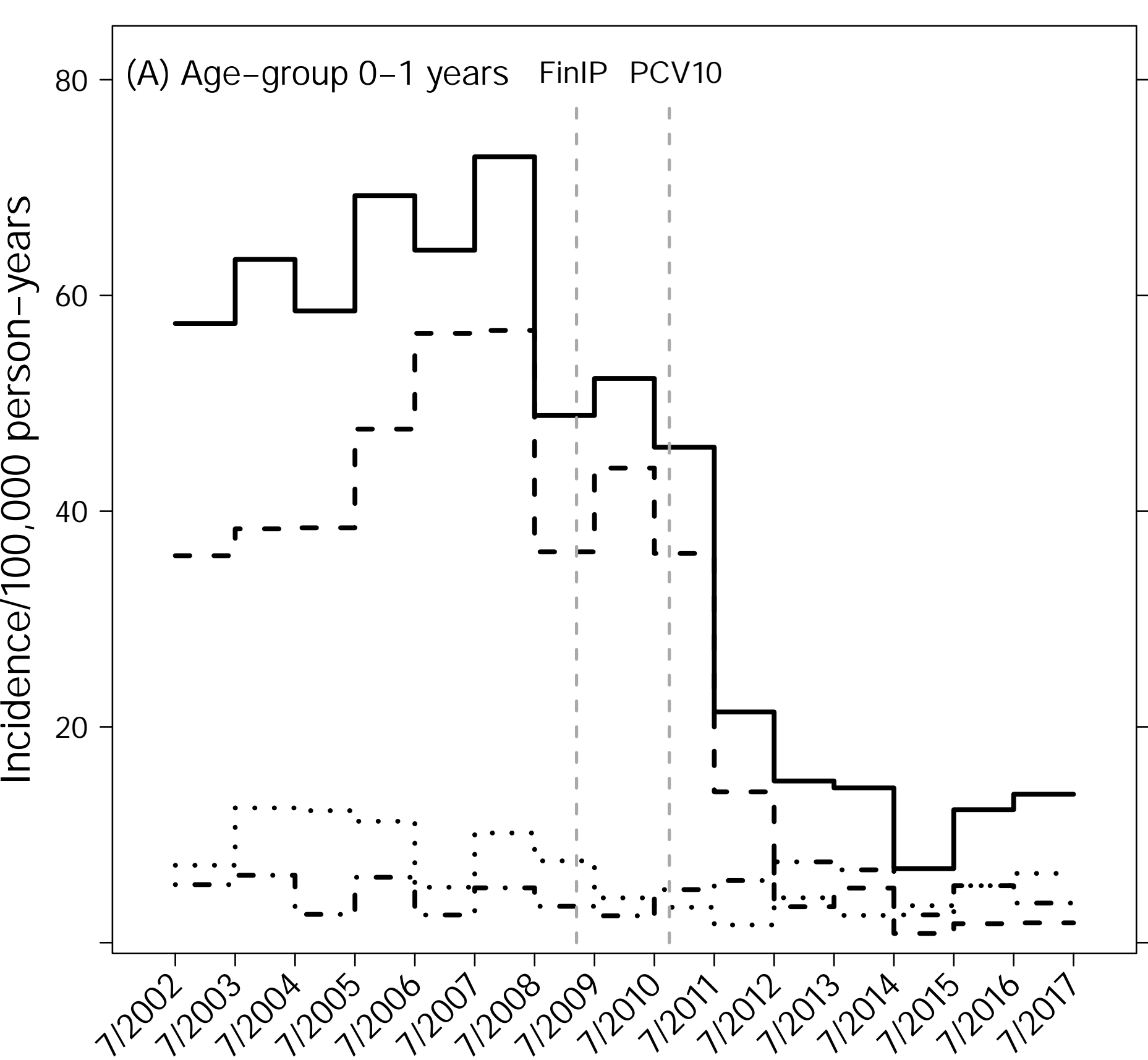

Epidemiological year

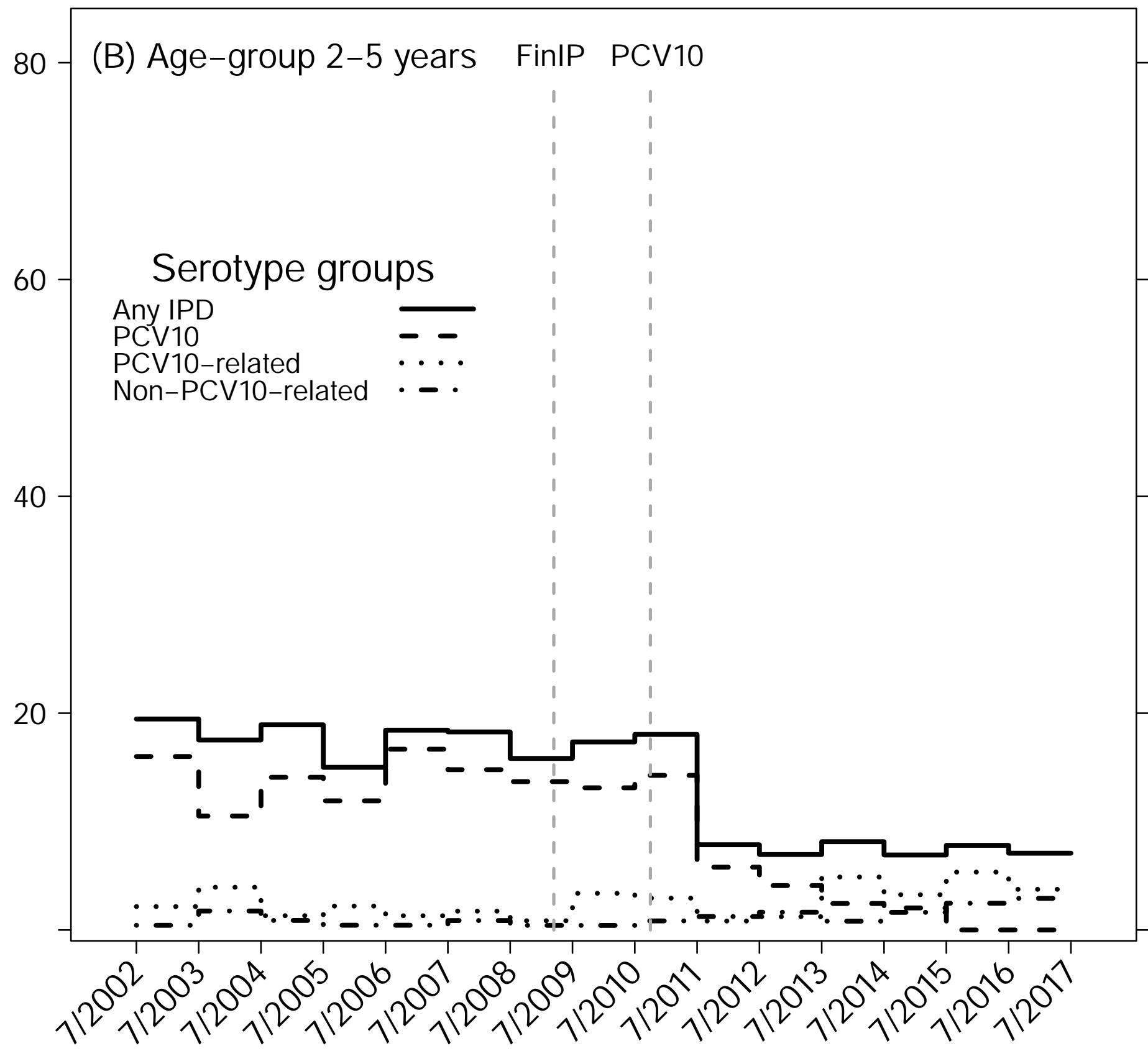

Epidemiological year 
(A) Age-group $0-1$ years FinIP PCV10

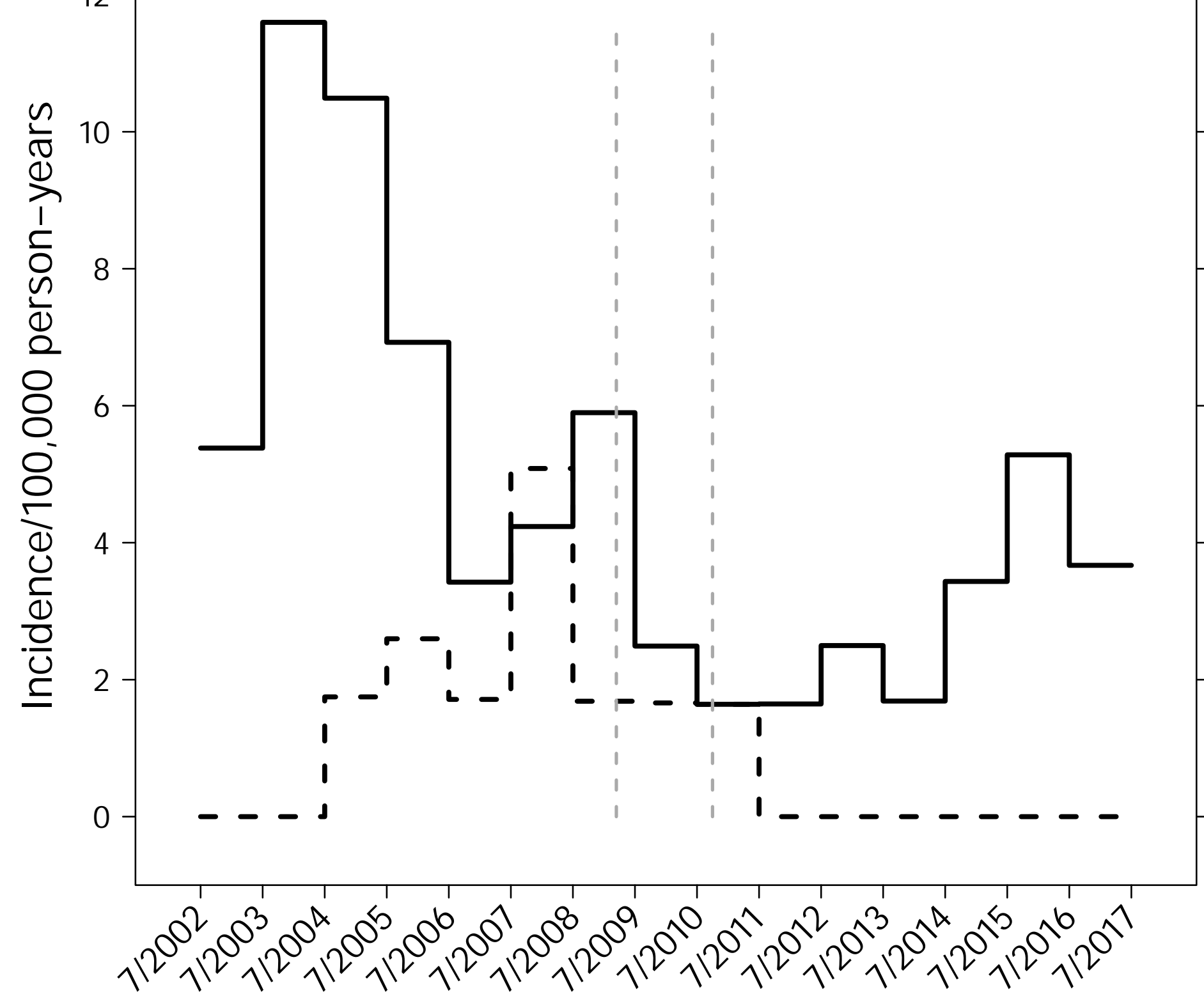

Epidemiological year
(B) Age-group 2-5 years FinlP PCV10

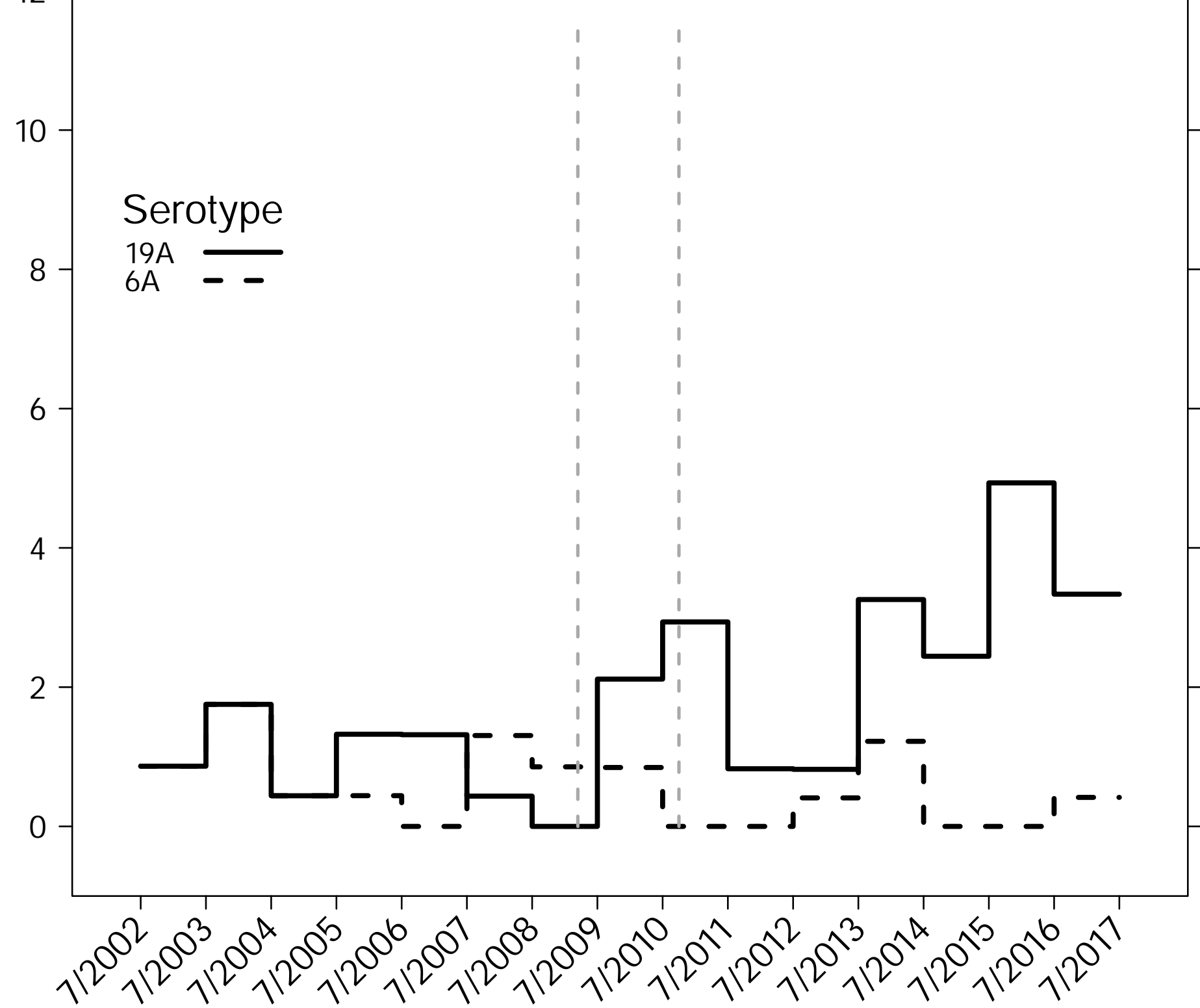

Epidemiological year 

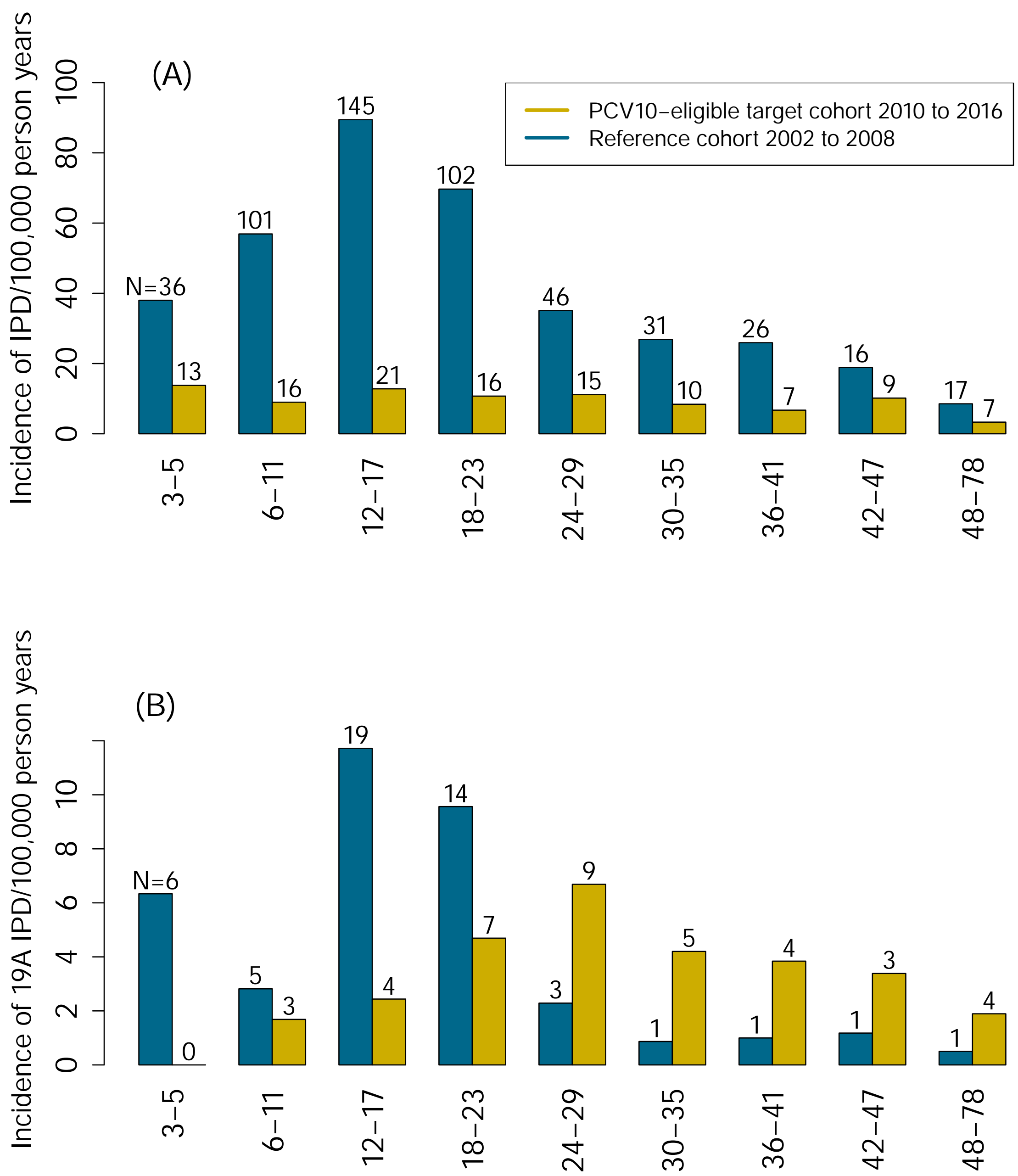
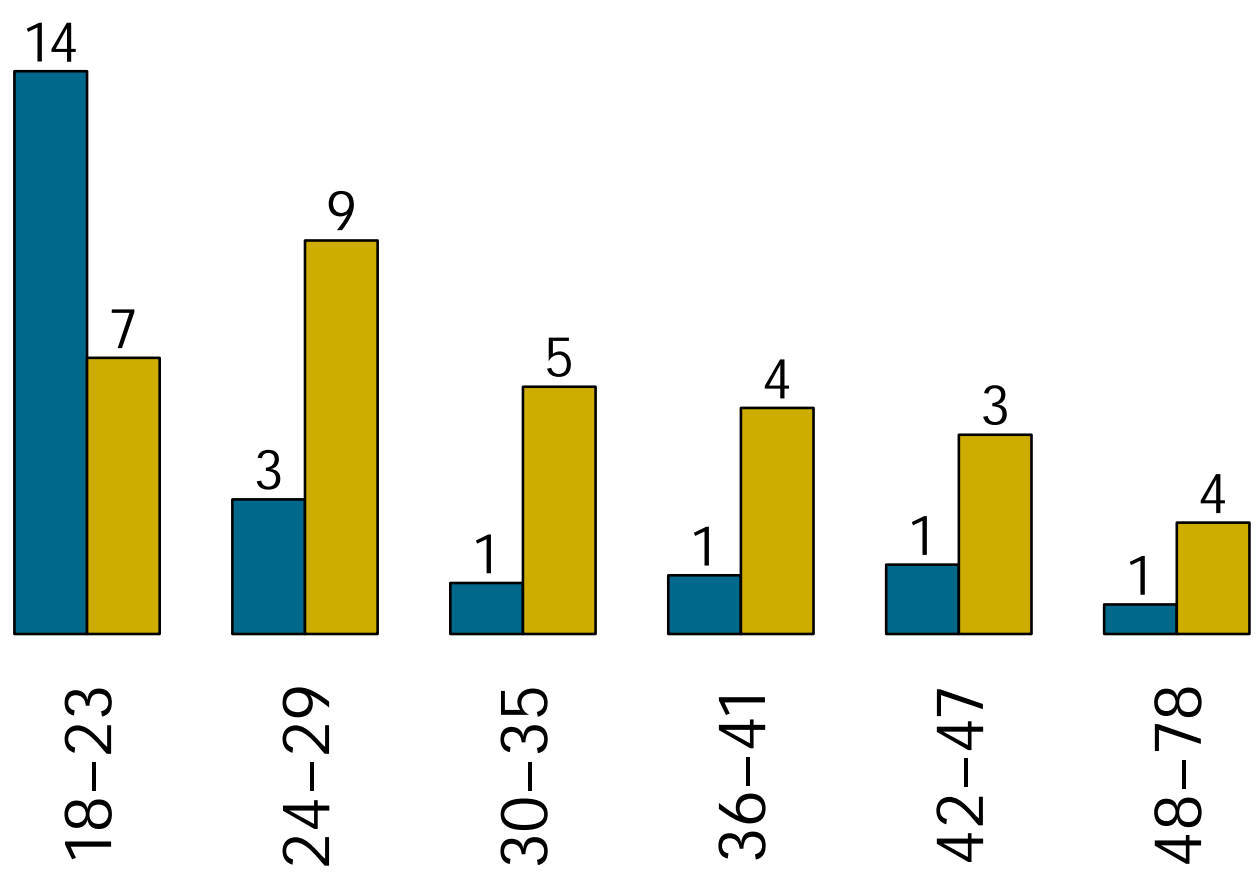

Age (months) 


\section{Supplementary Appendix}

Supplement to: Rinta-Kokko H, Palmu AA, Auranen K, et al. Long-term impact of 10-valent pneumococcal conjugate vaccination on invasive pneumococcal disease among children in Finland

Supplement Table 1. Incidence rates of invasive pneumococcal disease (IPD) of individual PCV10 serotypes and the corresponding relative and absolute incidence rate reductions, based on the comparison of the PCV10-eligible target cohort (2010-2016) vs. a reference cohort (2002-2008).

\begin{tabular}{|c|c|c|c|c|}
\hline \multirow[t]{2}{*}{ Serotype } & \multicolumn{2}{|c|}{ Incidence per 100,000 person-years (N) } & \multicolumn{2}{|c|}{ Target cohort vs. reference cohort } \\
\hline & $\begin{array}{l}\text { Reference cohort } \\
\text { 2002-2008* } \\
\text { Follow-up yrs } \\
1211504\end{array}$ & $\begin{array}{l}\text { Target cohort } \\
\text { 2010-2016** } \\
\text { Follow-up yrs } \\
1243145\end{array}$ & $\begin{array}{l}\text { Relative rate reduction, \% } \\
(95 \% \mathrm{Cl})\end{array}$ & $\begin{array}{l}\text { Absolute rate reduction per } \\
100,000 \text { person-years }(95 \% \mathrm{Cl})\end{array}$ \\
\hline 1 & $0.08(1)$ & $0.08(1)$ & $2.6(-2364.5,96.2)$ & $0(-0.2,0.2)$ \\
\hline 4 & $0.91(11)$ & $0(0)$ & $100(80.8,100)$ & $0.9(0.4,1.4)$ \\
\hline 5 & $0(0)$ & $0(0)$ & NA & $0(0,0)$ \\
\hline $6 B$ & $10.9(132)$ & $0.24(3)$ & $97.8(94.2,99.5)$ & $10.7(8.8,12.5)$ \\
\hline $7 F$ & $0.66(8)$ & $0.08(1)$ & $87.8(33.6,99.3)$ & $0.6(0.1,1.1)$ \\
\hline $9 \mathrm{~V}$ & $1.24(15)$ & $0(0)$ & $100(86.3,100)$ & $1.2(0.6,1.9)$ \\
\hline 14 & $8.58(104)$ & $0.64(8)$ & $92.5(85.6,96.6)$ & $7.9(6.2,9.7)$ \\
\hline $18 \mathrm{C}$ & $2.31(28)$ & $0.08(1)$ & $96.5(83.7,99.8)$ & $2.2(1.4,3.1)$ \\
\hline $19 F$ & $3.71(45)$ & $0.4(5)$ & $89.2(75.2,96.3)$ & $3.3(2.2,4.5)$ \\
\hline $23 \mathrm{~F}$ & $3.88(47)$ & $0.32(4)$ & $91.7(79.6,97.5)$ & $3.6(2.4,4.7)$ \\
\hline $6 \mathrm{~A}$ & 1.57 (19) & $0.08(1)$ & $94.9(75.3,99.7)$ & $1.5(0.8,2.2)$ \\
\hline $19 \mathrm{~A}$ & $4.21(51)$ & $3.14(39)$ & $25.5(-12.8,51.2)$ & $1.1(-0.5,2.6)$ \\
\hline
\end{tabular}

*Age 3-78 months, born Jun 2002 - Sep 2008

**Age 3-78 months, born Jun 2010 - Sep 2016

NA $=$ not applicable

Supplement Table 2. Incidence rates of invasive pneumococcal disease (IPD) of individual PCV10 serotypes and the corresponding relative and absolute incidence rate reductions, based on the comparison of an unvaccinated target cohort (2012-2016) vs. a reference cohort (2006-2010).

\begin{tabular}{|c|c|c|c|c|}
\hline \multirow[t]{2}{*}{ Serotype } & \multicolumn{2}{|c|}{ Incidence per 100,000 person-years $(\mathrm{N})$} & \multicolumn{2}{|c|}{ Unvaccinated target cohort vs. reference cohort } \\
\hline & $\begin{array}{l}\text { Reference cohort } \\
\text { 2006-2010* } \\
\text { Follow-up yrs } \\
1309618\end{array}$ & $\begin{array}{l}\text { Target cohort } \\
\text { 2012-2016** } \\
\text { Follow-up yrs } \\
1221933\end{array}$ & $\begin{array}{l}\text { Relative rate reduction, \% } \\
(95 \% \mathrm{Cl})\end{array}$ & $\begin{array}{l}\text { Absolute rate reduction per } \\
100,000 \text { person-years }(95 \% \mathrm{Cl})\end{array}$ \\
\hline 1 & $0(0)$ & $0(0)$ & NA & $0(0,0)$ \\
\hline 4 & $0.15(2)$ & $0(0)$ & $100(-66.5,100)$ & $0.2(-0.06,0.4)$ \\
\hline 5 & $0(0)$ & $0(0)$ & NA & $0(0,0)$ \\
\hline $6 \mathrm{~B}$ & $0.76(10)$ & $0.41(5)$ & $46.4(-50.8,83.3)$ & $0.4(-0.2,1.0)$ \\
\hline $7 F$ & $0.38(5)$ & $0.25(3)$ & $35.7(-162.1,86.8)$ & $0.1(-0.3,0.6)$ \\
\hline $9 \mathrm{~V}$ & $0(0)$ & $0.16(2)$ & $-\operatorname{Inf}(-\operatorname{lnf}, 40)$ & $-0.2(-0.4,0.06)$ \\
\hline 14 & $1.68(22)$ & $0.33(4)$ & $80.5(49.1,94.3)$ & $1.4(0.6,2.1)$ \\
\hline $18 \mathrm{C}$ & $1.3(17)$ & $0.49(6)$ & $62.2(9,86.4)$ & $0.8(0.08,1.5)$ \\
\hline $19 F$ & $0.46(6)$ & $0.16(2)$ & $64.3(-55.1,94.8)$ & $0.3(-0.1,0.7)$ \\
\hline $23 \mathrm{~F}$ & $1.15(15)$ & $0.65(8)$ & $42.8(-31.6,77)$ & $0.5(-0.3,1.2)$ \\
\hline $6 \mathrm{~A}$ & $0(0)$ & $0.25(3)$ & $-\operatorname{lnf}(-\operatorname{Inf},-7.3)$ & $-0.3(-0.5,0.03)$ \\
\hline $19 \mathrm{~A}$ & $0.69(9)$ & $0.74(9)$ & $-7.2(-174.5,58.2)$ & $-0.05(-0.7,0.6)$ \\
\hline
\end{tabular}

*Age 19-131 months, born Jan 2000 - May 2004

**Age 19-131 months, born Jan 2006 - May 2010

$\mathrm{NA}=$ not applicable 


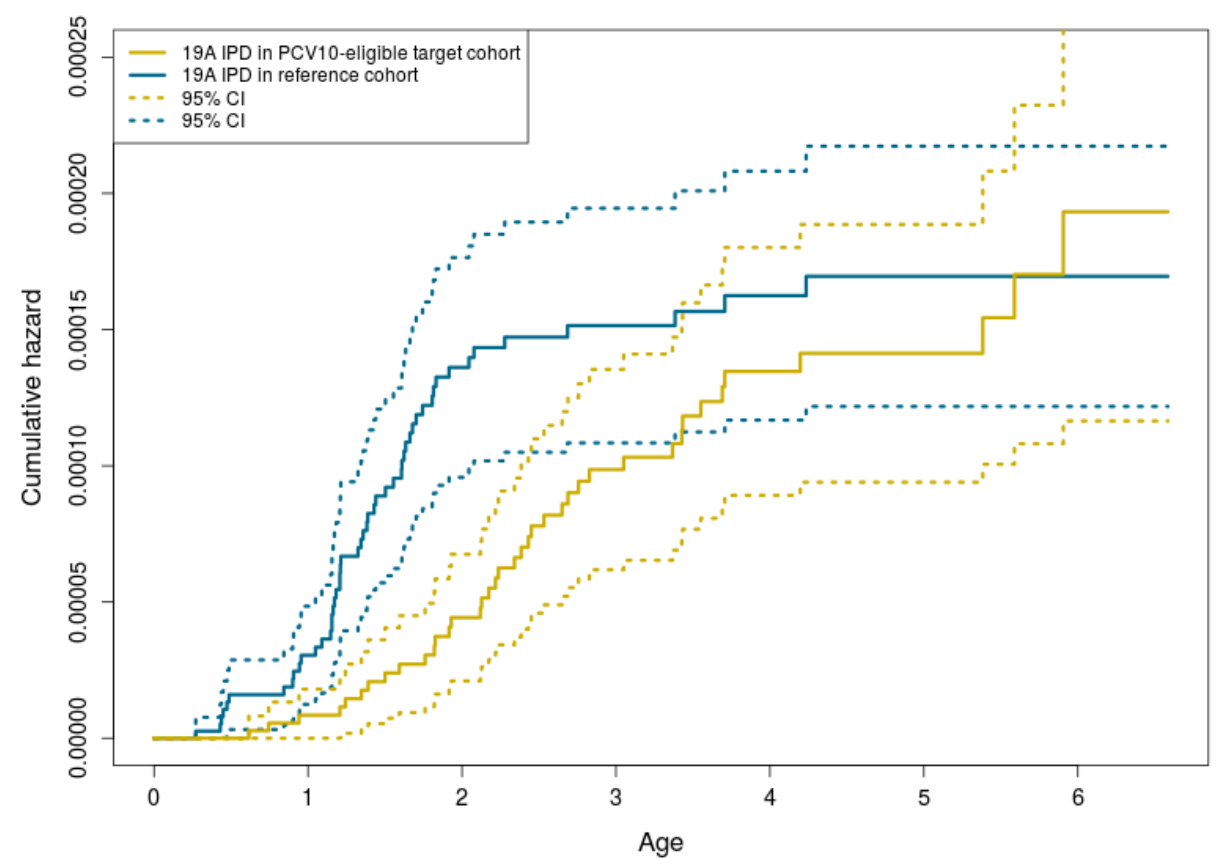

Supplement Figure 1. Age-specific cumulative hazards of 19A IPD in the PCV10-eligible target cohort and the reference cohort. 